\title{
Bending vibrations of stepped rods
}

\author{
Andrey Chernyaev \\ Department of Theoretical Mechanics, Orel State University, \\ Orel, Komsomolskaya Street, 95, 302026, Russia \\ E-mail: chernyev87@yandex.ru
}

Received 28 May 2017; accepted 29 May 2017

DOI https://doi.org/10.21595/vp.2017.18688

Check for updates

Abstract. Manufacturing stepped rods with segments of sizes proportional to stresses induced in operation is often used to reduce the material consumption in various fields of technology, for example, in the aircraft industry, where the requirements for the weight of structural elements are high. The vibration problems of continuous systems, i.e. systems which masses are considered distributed, are close to the resistance of materials and elasticity theory problems. They are described by partial differential equations. In this case, we consider a homogeneous isotropic material, obeying Hooke's law. Of all the vibration problems of continuous systems, the transverse vibration problems of shafts and beams is of greatest practical importance. The simplest examples of vibrations of prismatic rods were studied in the 18th century in works on acoustics. But before solving problems of practical importance, the problems of stepped beams, it had taken another two hundred years and the development of approximate methods of solving differential equations. The paper presents a solution to the problem of determining the fundamental frequencies of bending vibrations of two-stepped rods with various boundary conditions, using the approximate Lagrange-Ritz method. The calculation error does not exceed $2.6 \%$. The fundamental frequency of vibration is defined considering different lengths and stiffness ratios of stepped rod segments. The obtained results can be used in solving practical problems in various fields of technology.

Keywords: bending vibrations, stepped rod, fundamental frequency of vibration.

\section{Introduction}

A rod with a mass distributed along its length is considered as a system with an infinite number of degrees of freedom. The position of the rod at any time is determined by its elastic line, which is a function of two variables: the coordinates along the length $(x)$ and the time $(t)$. To determine the fundamental vibrations of such systems, the ratios of the theory of bending of rods, known from the discipline Resistance of materials, are used. There are exact solutions for plain rods with various types of supports. The exact solutions are obtained using so called "fundamental beam functions". For rods and rod systems, which elements have a variable cross-section, finding exact methods for calculating frequencies and principal modes of vibration is a very difficult problem. Therefore, it is urgent to develop and use approximate methods for solving such problems [1-10].

\section{Object and subject of research}

The research considers the bending vibrations of single-span stepped rods with the same and different types of supports at the ends: hinged at both ends, fixed at both ends, hinged at one end and fixed the other end. A stepped rod consists of several segments with dimensions taken in accordance with the ability to resist the stresses induced by loads (more stressed segments have a larger cross-section, less stressed ones have a smaller cross-section). A rod in bending is also called a beam. The fundamental frequency of vibration is defined considering different lengths and stiffness ratios of stepped rod segments. 


\section{Solution}

\subsection{A stepped rod fixed at both ends}

A rod consisting of three segments has a span $l$. The segments at supports have length $k l$ and stiffness $E I$, the middle segment has length (1-2k)l and stiffness $n E I$ (see Fig. 1).

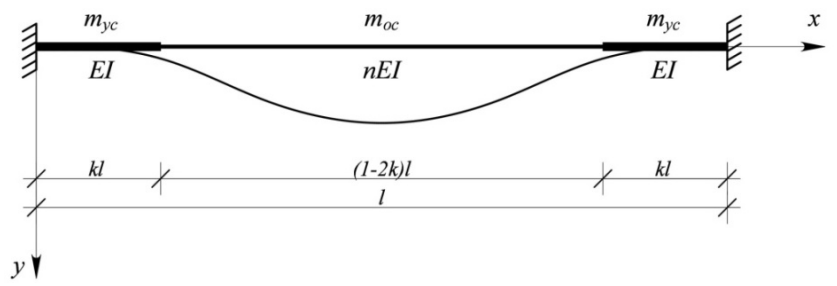

Fig. 1. A stepped rod fixed at both ends

To define the fundamental vibration frequency squared for a rod, the approximate Lagrange-Ritz method [10] can be used. In accordance with the method, the $k$ th vibration frequency squared can be defined by the following equation:

$\omega_{k}^{2}=\int_{0}^{l} E I(x)\left[\phi_{1}^{\prime \prime}(x)\right]^{2} d x / \int_{0}^{l} m(x) \phi_{1}^{2}(x) d x$,

where $\varphi_{1}(x)$ is a deflection function; $m(x)$ is the mass per unit length of a rod.

Let us take the deflection function of the rod in the given coordinate system as follows:

$\phi_{1}(x)=a\left(1-\cos \frac{2 \pi x}{l}\right)$

where $a$ - const.

The given function satisfies all boundary conditions. With all necessary transformations completed, taking into account the average value of mass per length unit, we obtain the following:

$\omega_{1}^{2}=\frac{64 E I \pi^{4}}{3 l^{4} \bar{m}} \cdot\left[\frac{1}{2}\left(k+\frac{\sin 4 \pi k}{4 \pi}\right)+n\left(\frac{1}{4}-\frac{1}{2}\left(k+\frac{\sin 4 \pi k}{4 \pi}\right)\right)\right]$,

where $\bar{m}=2\left(k \cdot m_{y c}+0,5(1-2 k) \cdot m_{o c}\right)-$ is the average mass per unit length.

By substituting the parameters $n=1$ and $k=0$ (or 0.5 ) into the Eq. (3), which corresponds to a plain rod, we obtain the following:

$\omega_{1}^{2}=\frac{(1,519 \pi)^{2}}{l^{2}} \cdot \sqrt{\frac{E I}{\bar{m}}}$

which differs from the exact solution [1]:

$\omega_{1}=\frac{(1,5 \pi)^{2}}{l^{2}} \cdot \sqrt{\frac{E I}{\bar{m}}}$ by $2.64 \%$.

Thus, the solution Eq. (3) gives acceptable result. 


\subsection{A stepped rod with hinged ends}

A rod consisting of three segments has a span $l$. The segments at supports have length $k l$ and stiffness $n E I$, the middle segment has length (1-2k)l and stiffness $E I$ (see Fig. 2).

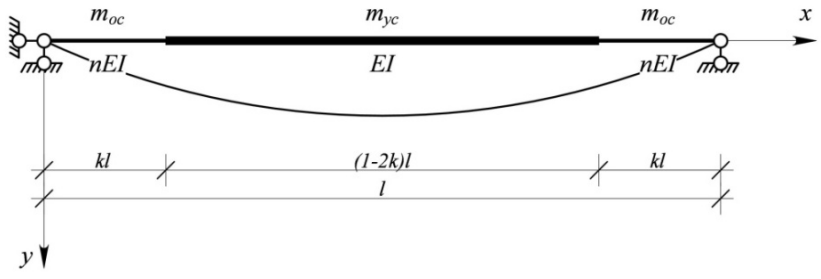

Fig. 2. A rod with hinged ends

Let us use the Lagrange-Ritz method. We take a deflection function as follows:

$\phi_{1}(x)=\sin \frac{\pi x}{l}$

The given function satisfies all boundary conditions. With all necessary transformations completed, taking into account the average value of mass per length unit, we obtain the following:

$\omega_{1}^{2}=\frac{4 E I \pi^{4}}{l^{4} \bar{m}} \cdot\left[\frac{1}{2}\left(k-\frac{\sin 2 \pi k}{2 \pi}\right) \cdot(n-1)+\frac{1}{4}\right]$,

where $\bar{m}=2\left(k \cdot m_{o c}+0,5(1-2 k) \cdot m_{y c}\right)-$ is the average mass per unit length.

By substituting the parameters $n=1$ and $k=0$ (or 0.5 ) into the Eq. (5), which corresponds to a plain rod, we obtain the exact solution [1]:

$\omega_{1}=\frac{\pi^{2}}{l^{2}} \cdot \sqrt{\frac{E I}{\bar{m}}}$.

\subsection{A stepped rod fixed at one end and hinged at the other end}

A rod consisting of two segments has a span $l$. The segment on the side with fixed end has length $k l$ and stiffness $E I$, the segment on the side with hinged end has length (1-k)l and stiffness $n E I$ (see Fig. 3).

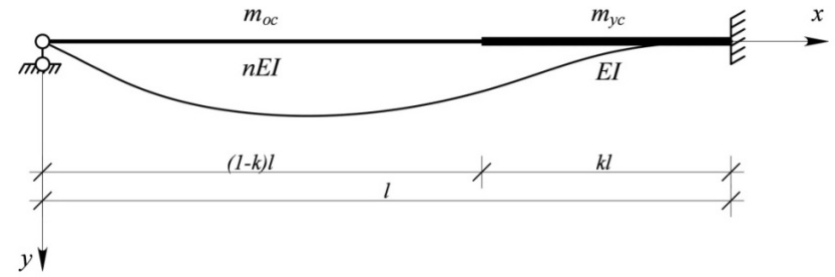

Fig. 3. A stepped rod fixed at one end and hinged at the other end

Let us use the Lagrange-Ritz method. For a deflection function we take the equation of the elastic line of a plain rod under uniformly distributed load $q$ along the entire length:

$\phi_{1}(x)=A \cdot\left(-l^{3} x+3 l x^{3}-2 x^{4}\right)$, 
where $A=q / 48 E I$.

The given function satisfies all boundary conditions. With all necessary transformations completed, taking into account the average value of mass per length unit, we obtain the following:

$\omega_{1}^{2}=\frac{630 E I}{19 l^{4} \bar{m}} \cdot\left[\left(108(1-k)^{3}-216(1-k)^{4}+115,2(1-k)^{5}\right) \cdot(n-1)+7,2\right]$,

where $\bar{m}=(1-k) \cdot m_{o c}+k \cdot m_{y c}$ is the average mass per unit length.

By substituting the parameters $n=1$ and $k=0$ (or 1) into the Eq. (7), which corresponds to a plain rod, with number $\pi$ intentionally introduced, we obtain the following:

$\omega_{1}=\frac{(1,251 \pi)^{2}}{l^{2}} \cdot \sqrt{\frac{E I}{\bar{m}}}$.

Which differs from the exact solution [1]:

$\omega_{1}^{2}=\frac{(1,25 \pi)^{2}}{l^{2}} \cdot \sqrt{\frac{E I}{\bar{m}}}$ by $0.3 \%$.

Thus, the solution Eq. (7) gives acceptable result.

\section{Numerical investigations}

Let us define the influence of the lengths and varying stiffness ratios of a stepped rod on its fundamental frequency of vibration. A steel stepped rod with different types of supports at its ends has the following parameters: $\operatorname{span} l=6 \mathrm{~m}$, the smaller cross-section dimensions of the rod is $h \times b=16 \times 5 \mathrm{~cm}$ (second moment of area $I_{x}=1707 \mathrm{~cm}^{4}$ ), the larger cross-section dimensions of the rod is $h \times b=16 \times 8 \mathrm{~cm}$ (second moment of area $I_{x}=2731 \mathrm{~cm}^{4}$ ), elastic modulus $E=2 \cdot 10^{5} \mathrm{MPa}$.

A parameter $n=1707 / 2731=0.625$. Mass per unit length for the segment of smaller cross-section is $m_{o c}=0.05 \cdot 0.16 \cdot 1 \cdot 7850=62.8 \mathrm{~kg} / \mathrm{m}$ and for the segment of larger cross-section is $m_{y c}=0.08 \cdot 0.16 \cdot 1 \cdot 7850=100.48 \mathrm{~kg} / \mathrm{m}$. The parameter $k$ is varying in such way that a plain rod with $n E I$ stiffness turns into a stepped rod with $n E I$ and $E I$ stiffness, and then into a plain rod with $E I$ stiffness. Equivalent mass of all three rods increases proportionally. The calculation is performed using the obtained Eqs. (3), (5), (7). Results of the calculation are shown in Table 1 and Fig. 4.

Table 1. Results of numerical study of stepped rods with various types of supports

\begin{tabular}{|c|c|c|c|c|c|c|}
\hline $\begin{array}{c}\text { Type of } \\
\text { supports }\end{array}$ & \multicolumn{2}{|c|}{ Fixed at both ends (Fig. 1) } & \multicolumn{2}{|c|}{ Hinged at both ends (Fig. 2) } & \multicolumn{2}{|c|}{$\begin{array}{c}\text { Fixed at one end and hinged } \\
\text { at the other end (Fig. 3) }\end{array}$} \\
\hline $\begin{array}{c}\text { Mass } m, \\
\mathrm{~kg} / \mathrm{m}\end{array}$ & $k$ & $\begin{array}{c}\text { Vibration frequency } \omega, \\
\text { sec-1 according to Eq. (3) }\end{array}$ & $k$ & $\begin{array}{c}\text { Vibration frequency } \omega, \\
\text { sec-1 according to Eq. (5) }\end{array}$ & $k$ & $\begin{array}{c}\text { Vibration frequency } \omega, \\
\text { sec-1 according to Eq. (7) }\end{array}$ \\
\hline 62.8 & 0 & 143.824 & 0.5 & 63.922 & 0 & 99.777 \\
\hline 70.336 & 0.1 & 121.344 & 0.4 & 53.007 & 0.2 & 82.538 \\
\hline 77.872 & 0.2 & 119.317 & 0.3 & 54.109 & 0.4 & 79.187 \\
\hline 81.64 & 0.25 & 116.705 & 0.25 & 54.119 & 0.5 & 79.113 \\
\hline 85.408 & 0.3 & 114.271 & 0.2 & 53.803 & 0.6 & 79.88 \\
\hline 92.944 & 0.4 & 113.067 & 0.1 & 52.416 & 0.8 & 81.06 \\
\hline 100.48 & 0.5 & 143.819 & 0 & 63.919 & 1 & 99.773 \\
\hline
\end{tabular}




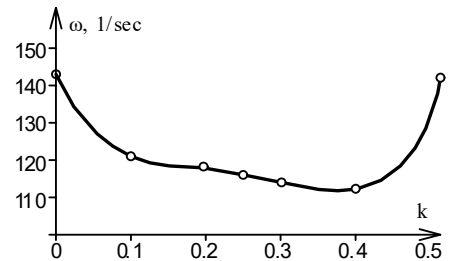

a) Fig. 1

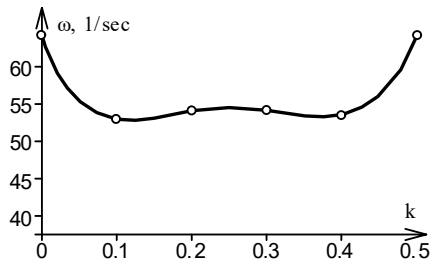

b) Fig. 2

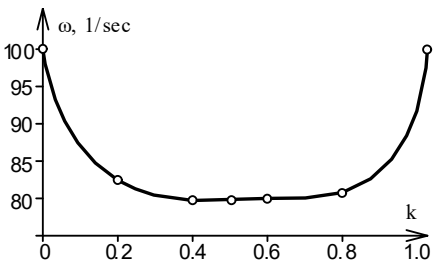

c) Fig. 3

Fig. 4. Vibration frequency curves for rods of various stiffness with various types of supports

\section{Conclusions}

Using the Lagrange-Ritz method, there have been obtained some approximate solutions to the problem of bending vibrations of stepped rods with various types of supports. The calculation error does not exceed $2.6 \%$, which is accurate enough for engineering calculations.

Numerical studies have shown the influence of the lengths and varying stiffness ratios of a stepped rod on its fundamental frequency of vibration.

\section{References}

[1] Gordon V., Stepanov Y., Shmarkova L. Frequencies of own bend vibrations of perforated bars. Proceedings of the 10th International Congress on Sound and Vibration, 2003, p. 469-476.

[2] Gordon V., Stepanov Yu, Anokhin P. Determination of stiffness properties of a heterogeneous beam by it's dynamical characteristics. Proceedings of the 2nd International Conference on Heterogeneous Material Mechanics, 2008, p. 1381-1382.

[3] Andrianov I., Markert B., Awrejcewicz J., Danishevskyy V. Influence of geometric and physical nonlinearities on the internal resonances of a finite continuous rod with a microstructure. Journal of Sound and Vibration, Vol. 386, 2017, p. 359-371.

[4] Kaplunov J., Prikazchikov D., Sergushova O. Multi-parametric analysis of the lowest natural frequencies of strongly inhomogeneous elastic rods. Journal of Sound and Vibration, Vol. 366, 2016, p. $264-276$.

[5] Gan C., Wei Y., Yang S. Longitudinal wave propagation in a multi-step rod with variable cross-section. Journal of Vibration and Control, Vol. 22, Issue 3, 2016, p. 837-852.

[6] Eisenberger M. Exact longitudinal vibration frequencies of a variable cross-section rod. Applied Acoustics, Vol. 34, Issue 2, 1991, p. 123-130.

[7] Hsueh W.-J. Free and forced vibrations of stepped rods and coupled systems. Journal of Sound and Vibration, Vol. 226, Issue 5, 1999, p. 891-904.

[8] Li Q. S. Exact solutions for free longitudinal vibration of stepped non-uniform rods. Applied Acoustics, Vol. 60, Issue 1, 2000, p. 13-28.

[9] Zak A., Krawczuk M. Certain numerical issues of wave propagation modelling in rods by the spectral finite element method. Finite Elements in Analysis and Design, Vol. 47, Issue 9, 2011, p. 1036-1046.

[10] Leissa A. W. The historical bases of the Rayleigh and Ritz methods. Journal of Sound and Vibration, Vol. 287, Issue 4, 2005, p. 961-978. 